Pacific Journal of Mathematics 


\section{FITTING STRUCTURES}

\section{Karsten Johnsen and HaRTMUt LaUe}

Motivated by papers of $\mathrm{H}$. Fitting, the problem arises whether there exists a ring which contains a given ring and a semigroup acting on each other. This problem is solved in the affirmative by the construction of a "universal envelopment". Furthermore, the situation investigated gives rise to a generalized wreath product which is used for a description of certain automorphism groups.

0. Introduction. The endomorphisms of an abelian group form a ring in a natural and well-known way, whereas in the case of a nonabelian group one has no general "addition" of endomorphisms. One easily proves that the "sum" of two endomorphisms $\alpha, \beta$ of a group $G$,

$$
\alpha+\beta: g \mapsto g^{\alpha} g^{\beta} \quad \text { for all } g \in G,
$$

is an endomorphism of $G$ if and only if $\left[G^{\alpha}, G^{\beta}\right]=1$. Hence the endomorphisms that can be added to any endomorphism are exactly the homomorphisms of $G$ into its center $Z(G)$. In this sense, the ring $\operatorname{Hom}(G, Z(G))$ is a "pleasant" substructure of $\operatorname{End}(G)$. Long ago, Fitting [2] described the structure of $\operatorname{End}_{G}(G)$ which, though not a ring, still has numerous ring properties; if we put $H:=\operatorname{End}_{G}(G), S:=\operatorname{Hom}(G, Z(G))$, then e.g.

$$
\begin{aligned}
& \left(s_{1} h\right) s_{2}=s_{1}\left(h s_{2}\right) \quad \text { for all } h \in H, s_{1}, s_{2} \in S \text {, } \\
& \left(h_{1}+s\right) h_{2}=h_{1} h_{2}+s h_{2}, \quad h_{1}\left(h_{2}+s\right)=h_{1} h_{2}+h_{1} s \\
& \text { for all } h_{1}, h_{2} \in H, s \in S \text {, } \\
& \left(h+s_{1}\right) s_{2}=h s_{2}+s_{1} s_{2}, \quad s_{1}\left(h+s_{2}\right)=s_{1} h+s_{1} s_{2} \\
& \text { for all } h \in H, s_{1}, s_{2} \in S \text {. }
\end{aligned}
$$

Keeping these rules as axioms, we introduce so-called Fitting structures in the first chapter of this paper and show that firstly there does exist a ring $R$ containing $H$ and $S$ such that (1), (2), (3) are special cases of its associative and distributive laws, and that secondly any ring with this property (if - which is a non-essential restriction-it is generated by $H$ ) is a homomorphic image of $R$. Emanating naturally from Fitting's notion of "Bereich" [2], the problem of the existence of enveloping rings for Fitting structures, which has been solved for $H=\operatorname{End}_{G}(G)$ by Fitting 
in a special way, thus finds a general positive answer. Chapter 2 shows that even if there is not given an addition of elements of $H$ and $S$, such an addition can be defined after a certain enlargement of $H$, and that this process of making $H$ and $S$ into a Fitting structure is essentially uniquely determined by the actions of $H$ on $S$. In Chapter 3, we introduce to each Fitting structure a generalized wreath product containing the usual wreath product of (semi-)groups as a special case, and use this concept to give a simple description of certain automorphism groups, applying a result of another paper by Fitting [1].

1. Fitting structures. For every ring ${ }^{1} S$, we put

$\operatorname{End}_{\Lambda}(S):=\left\{\alpha \mid \alpha \in \operatorname{End}(S,+),\left(s_{1} s_{2}\right)^{\alpha}=s_{1}\left(s_{2}^{\alpha}\right)\right.$ for all $\left.s_{1}, s_{2} \in S\right\}$,

$\operatorname{End}_{\mathrm{P}}(S):=\left\{\alpha \mid \alpha \in \operatorname{End}(S,+),\left(s_{1} s_{2}\right)^{\alpha}=\left(s_{1}^{\alpha}\right) s_{2}\right.$ for all $\left.s_{1}, s_{2} \in S\right\}$.

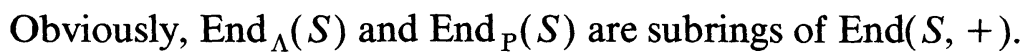

1.1. Definition. Let $H$ be a semigroup, $S$ a ring, $\varphi$ a homomorphism of $H$ into the multiplicative semigroup of $\operatorname{End}_{\Lambda}(S), \psi$ an antihomomorphism of $H$ into the multiplicative semigroup of $\operatorname{End}_{\mathrm{P}}(S)$ such that $H^{\varphi}$ and $H^{\psi}$ commute elementwise. Let $\sigma$ be a homomorphism of $(S,+)$ into the symmetric group $\mathfrak{\Im}_{H}$ on $H$ such that $h^{s^{\sigma}}=h$ implies $s=0$ for all $h \in H, s \in S$ (i.e., $(S,+)$ "acts freely" on $H$ ).

If $h \in H, s \in S$, we write $s h$ for $s^{h^{\varphi}}, h s$ for $s^{h^{\psi}}, h+s$ for $h^{s^{\sigma}}$. The 5-tuple $(H, S, \varphi, \psi, \sigma)$ is called a Fitting structure if (1), (2), (3) hold. ${ }^{2}$

By definition, we have

$$
\begin{array}{cc}
\left(s h_{1}\right) h_{2}=s\left(h_{1} h_{2}\right), & h_{1}\left(h_{2} s\right)=\left(h_{1} h_{2}\right) s \\
& \text { for all } h_{1}, h_{2} \in H, s \in S, \\
\left(h+s_{1}\right)+s_{2}=h+\left(s_{1}+s_{2}\right) & \text { for all } h \in H, s_{1}, s_{2} \in S, \\
\left(s_{1} s_{2}\right) h=s_{1}\left(s_{2} h\right), & h\left(s_{1} s_{2}\right)=\left(h s_{1}\right) s_{2} \\
& \text { for all } h \in H, s_{1}, s_{2} \in S, \\
\left(h_{1} s\right) h_{2}=h_{1}\left(s h_{2}\right) \quad \text { for all } h_{1}, h_{2} \in H, s \in S & \text { for all } h \in H, s \in S .
\end{array}
$$

${ }^{1}$ All rings in this paper are associative, but do not necessarily have an identity element.

${ }^{2}$ If $H$ is a "Bereich" in the sense of Fitting [2], let $S$ be the set of all elements of $H$ which can be added to any element of $H$. Then $S$ is a ring, and we get a Fitting structure with the additional property that $S$ is contained in $H$, and $H$ has an identity element. Any further possibilities to add elements of $H$ (which might exist in Fitting's "Bereich") are treated as non-existent in our Fitting structures. 
If $H$ has an identity element 1 , then (2) and (8) imply $1^{\varphi}=\mathrm{id}=1^{\psi}$.

Fitting structures $\mathscr{F}=(H, S, \varphi, \psi, \sigma), \mathscr{F}^{\prime}=\left(H^{\prime}, S^{\prime}, \varphi^{\prime}, \psi^{\prime}, \sigma^{\prime}\right)$ are called isomorphic if there are isomorphisms $\alpha$ of $H$ onto $H^{\prime}, \beta$ of $S$ onto $S^{\prime}$ with the properties $\beta \sigma^{\prime}=\sigma \bar{\alpha}, \alpha \varphi^{\prime}=\varphi \bar{\beta}, \alpha \psi^{\prime}=\psi \bar{\beta}$, where $\bar{\alpha}$ is the isomorphism of $\Im_{H}$ onto $\Im_{H^{\prime}}$ induced by $\alpha$ (such that $\pi^{\bar{\alpha}}=\alpha^{-1} \pi \alpha$ for all $\left.\pi \in \mathbb{S}_{H}\right)$, and $\bar{\beta}$ is the isomorphism of $\operatorname{End}(S)$ onto $\operatorname{End}\left(S^{\prime}\right)$ induced by $\beta$ (such that $\zeta^{\bar{\beta}}=\beta^{-1} \zeta \beta$ for all $\zeta \in \operatorname{End}(S)$ ).

$\mathscr{F}^{\prime}$ is called a Fitting substructure of $\mathscr{F}$ if $H^{\prime}$ is a subsemigroup of $H, S^{\prime}$ is a subring of $S$, and $\varphi^{\prime}=\left.\varphi\right|_{H^{\prime}}, \psi^{\prime}=\left.\psi\right|_{H^{\prime}}, \sigma^{\prime}=\left.\sigma\right|_{S^{\prime}}$. If $H^{\prime}$ is a subsemigroup of $H, S^{\prime}$ a subring of $S$, then $\left(H^{\prime}, S^{\prime},\left.\varphi\right|_{H^{\prime}},\left.\psi\right|_{H^{\prime}},\left.\sigma\right|_{S^{\prime}}\right)$ is a Fitting substructure of $\mathscr{F}$ if and only if $S^{\prime} H^{\prime} \subseteq S^{\prime}, H^{\prime} S^{\prime} \subseteq S^{\prime}$, and $H^{\prime}+S^{\prime}=H^{\prime}$.

1.2. Definition. Let $\mathscr{F}$ be a Fitting structure, $R$ a ring, $\sim$ a homomorphism of $H$ into the multiplicative semigroup of $R$, and ${ }^{-}$a homomorphism of $S$ onto an ideal of $R$. The triple $\left(R, \sim,{ }^{-}\right)$is called an envelopment of $\mathscr{F}$ if

$$
\tilde{h}+\bar{s}=\widetilde{h+s} \text { for all } h \in H, s \in S
$$

holds.

The envelopment $\left(R,^{\sim},-\right)$ is called faithful if ${ }^{\sim}$ and ${ }^{-}$are injective.

From (9), we conclude

$$
\tilde{h} \bar{s}=\bar{h} s, \bar{s} \tilde{h}=\overline{s h} \quad \text { for all } h \in H, s \in S,
$$

since

$$
\widetilde{h^{2}}+\overline{s h}=\widetilde{h^{2}+s h}=\widetilde{(h+s) h}=\widetilde{h+s \tilde{h}}=(\tilde{h}+\bar{s}) \tilde{h}=\widetilde{h^{2}}+\bar{s} \tilde{h},
$$

and the second part of $(10)$ is proved similarly.

1.3. Definition. Let $\mathscr{F}$ be a Fitting structure and $\left(R, \sim,-,\left(R^{\prime}, \stackrel{\sim}{,}\right)\right.$ envelopments of $\underset{\sim}{\mathscr{F}}$. Then a mapping $\chi$ is called a homomorphism of $(R, \stackrel{\sim}{,})$ into $\left(R^{\prime}, \stackrel{\approx}{=}\right)$ if $\chi$ is a homomorphism of $R$ into $R^{\prime}$ such that $\tilde{h}^{\chi}=\tilde{h}, \bar{s}^{\chi}=\overline{\bar{s}}$ for all $h \in H, s \in S$. If $\chi$ is an isomorphism of $R$ onto $R^{\prime}$, we call our envelopments isomorphic. An envelopment $\mathscr{U}$ of $\mathscr{F}$ is called universal if for any envelopment $\mathscr{V}$ of $\mathscr{F}$ there is a homomorphism of $\mathscr{U}$ into $\mathscr{V}$.

Universal envelopments of isomorphic Fitting structures are isomorphic. We now prove the following existence theorem:

1.4. THEOREM. Every Fitting structure has a faithful universal envelopment. 
Proof. Let $\mathscr{F}=(H, S, \varphi, \psi, \sigma)$ be the Fitting structure given. We put $T:=\mathbb{Z} H$ and write $\hat{+}, \hat{\cdot}$ for the standard addition and multiplication in $T$. (Then $h_{1} \hat{\imath} h_{2}=h_{1} h_{2}$ for all $h_{1}, h_{2} \in H$.) For $h \in H, s \in S$ define

$$
\delta(h, s):=(h+s) \wedge h
$$

and let $K$ be the additive subgroup of $T$ generated by $\left\{\delta\left(h_{1}, s\right) \hat{-}\right.$ $\left.\delta\left(h_{2}, s\right) \mid h_{1}, h_{2} \in H, s \in S\right\}$. Then

$$
\begin{aligned}
h_{1} \hat{\circ} \delta\left(h_{2}, s\right) \hat{\sim} \delta\left(h_{2}, h_{1} s\right), \delta\left(h_{2}, s\right) \hat{h_{1}} \hat{\sim} \delta\left(h_{2}, s h_{1}\right) \in K \\
\\
\text { for all } h_{1}, h_{2} \in H, s \in S,
\end{aligned}
$$

as

$$
\begin{aligned}
h_{1} \hat{\circ}\left(h_{2}, s\right) \hat{\sim} \delta\left(h_{2}, h_{1} s\right) & =h_{1} \hat{\bullet}\left(\left(h_{2}+s\right) \hat{\sim} h_{2}\right) \hat{\sim} \delta\left(h_{2}, h_{1} s\right) \\
& =h_{1}\left(h_{2}+s\right) \hat{\sim} h_{1} h_{2} \hat{\sim} \delta\left(h_{2}, h_{1} s\right) \\
& =\delta\left(h_{1} h_{2}, h_{1} s\right) \hat{\sim} \delta\left(h_{2}, h_{1} s\right) \in K,
\end{aligned}
$$

the second part of (11) being proved analogously. Obviously, (11) yields

$$
\begin{aligned}
h_{1} \hat{\bullet}\left(\delta\left(h_{2}, s\right) \hat{-\delta}\left(h_{3}, s\right)\right),\left(\delta\left(h_{2}, s\right) \hat{\sim} \delta\left(h_{3}, s\right)\right) \hat{*} h_{1} & \in K \\
& \text { for all } h_{1}, h_{2}, h_{3} \in H, s \in S .
\end{aligned}
$$

Thus $K$ is an ideal of $T$, and, by definition of $K$,

$$
\delta\left(h_{1}, s\right) \hat{+} K=\delta\left(h_{2}, s\right) \hat{+} K \quad \text { for all } h_{1}, h_{2} \in H, s \in S \text {. }
$$

We now define

$$
\begin{aligned}
R: & =T / K, \\
\sim & \rightarrow R, h \mapsto h \hat{+} K,
\end{aligned}
$$

and for an arbitrary $h \in H$

$$
-: S \rightarrow R, \quad s \mapsto \delta(h, s) \hat{+} K .
$$

(By (13), ${ }^{-}$is independent of the choice of $h$.) Obviously, $\sim$ is a homomorphism of $H$ into the multiplicative semigroup of $R$, and

$$
\begin{aligned}
\widetilde{h+s} & =(h+s) \hat{+} K=h \hat{+}(h+s) \hat{-} h \hat{+} K \\
& =(h \hat{+} K) \hat{+}(\delta(h, s) \hat{+} K)=\tilde{h} \hat{+} \bar{s} \quad \text { for all } h \in H, s \in S,
\end{aligned}
$$

whence (9) holds. We want to show that ${ }^{-}$is a ring homomorphism of $S$ into an ideal of $R$, and start with

(14) $\delta\left(h, s_{1}+s_{2}\right) \wedge \delta\left(h, s_{1}\right) \wedge \delta\left(h, s_{2}\right) \in K \quad$ for all $h \in H, s_{1}, s_{2} \in S$.

For

$$
\begin{aligned}
\delta(h, & \left.s_{1}+s_{2}\right) \hat{\sim} \delta\left(h, s_{1}\right) \hat{\sim} \delta\left(h, s_{2}\right) \\
& =\left(h+s_{1}+s_{2}\right) \hat{\sim} h \hat{\sim}\left(h+s_{1}\right) \hat{+} h \hat{\sim} \delta\left(h, s_{2}\right) \\
& =\delta\left(h+s_{1}, s_{2}\right) \wedge \delta\left(h, s_{2}\right) \in K .
\end{aligned}
$$


Furthermore,

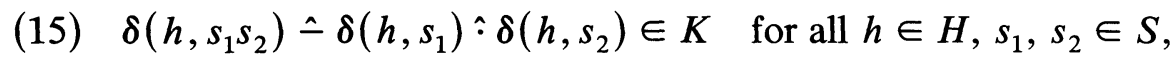

since

$$
\begin{aligned}
& \delta\left(h, s_{1} s_{2}\right) \wedge \delta\left(h, s_{1}\right) \div \delta\left(h, s_{2}\right) \\
& =\delta\left(h, s_{1} s_{2}\right) \hat{\sim}\left(\left(h+s_{1}\right) \hat{\sim} h\right) \hat{\bullet}\left(\left(h+s_{2}\right) \hat{\sim} h\right) \\
& =\delta\left(h, s_{1} s_{2}\right) \hat{\sim}\left(h+s_{1}\right)\left(h+s_{2}\right) \hat{+}\left(h+s_{1}\right) h \hat{+} h\left(h+s_{2}\right) \hat{-} h^{2} \\
& =\delta\left(h, s_{1} s_{2}\right) \hat{\sim} \delta\left(\left(h+s_{1}\right) h,\left(h+s_{1}\right) s_{2}\right) \hat{+} \delta\left(h^{2}, h s_{2}\right) \in K,
\end{aligned}
$$

by (13) and (14).

As (14) and (15) show, ${ }^{-}$is a ring homomorphism, and by (12), $\bar{S}$ is an ideal of $R$. Therefore, $\left(R, \sim^{-}\right)$is an envelopment of $\mathscr{F}$. We need some preliminaries to show that ${ }^{-}$and are injective:

Let $D$ be the additive subgroup of $T$ generated by $\{\delta(h, s) \mid h \in H$, $s \in S\}$. Then $K \leq D$, and $D / K=\bar{S}$. Since

$$
\hat{-} \delta(h, s)=\delta(h+s,-s) \text { for all } h \in H, s \in S,
$$

every element of $D$ has the form $\sum_{j} \delta\left(h_{j}, s_{j}\right)$ for appropriate $h_{j} \in H$, $s_{j} \in S$. We claim:

$$
\sum_{j=1}^{k} \delta\left(h_{j}, s_{j}\right)=0 \Rightarrow \sum_{j=1}^{k} s_{j}=0
$$

for all $h_{1}, \ldots, h_{k} \in H, s_{1}, \ldots, s_{k} \in S$.

Suppose $\sum_{j=1}^{k} \delta\left(h_{j}, s_{j}\right)=0$. Then we have $\sum_{j=1}^{k}\left(h_{j}+s_{j}\right)=\sum_{j=1}^{k} h_{j}$. Since $(T, \hat{+})$ is free over $H$, there is a permutation $\pi$ of $\{1, \ldots, k\}$ such that $h_{j}+s_{j}=h_{j \pi}$ for all $j \in\{1, \ldots, k\}$. For $i \in\{1, \ldots, k\}$, let $f_{i}$ be the smallest positive integer such that $i \pi^{f_{i}}=i$. Then $\left\{i, i \pi, \ldots, i \pi^{f_{i}-1}\right\}$ is the orbit of $i$ under $\pi$, and $h_{i}+s_{i}+s_{i \pi}+\cdots+s_{i \pi^{l}}=h_{i \pi^{l+1}}$ for $0 \leq l<f_{i}$, hence in particular $h_{i}+s_{i}+s_{i \pi}+\cdots+s_{i \pi^{f_{t}-1}}=h_{i}$, and $s_{i}+s_{i \pi}$ $+\cdots+s_{i \pi^{f_{i}-1}}=0$ by (8). Now if $X$ denotes a full set of representatives of the orbits of $\pi$ in $\{1, \ldots, k\}$,

$$
\sum_{j=1}^{k} s_{j}=\sum_{i \in X}\left(s_{i}+s_{i \pi}+\cdots+s_{i \pi^{f_{i}-1}}\right)=0,
$$

proving (17).

As a consequence, we have

$$
\sum_{j=1}^{k} \delta\left(h_{j}, s_{j}\right)=\sum_{j=1}^{k^{\prime}} \delta\left(h_{j}^{\prime}, s_{j}^{\prime}\right) \Rightarrow \sum_{j=1}^{k} s_{j}=\sum_{j=1}^{k^{\prime}} s_{j}^{\prime}
$$

for all $h_{1}, \ldots, h_{k}, h_{1}^{\prime}, \ldots, h_{k^{\prime}}^{\prime} \in H, s_{1}, \ldots, s_{k}, s_{1}^{\prime}, \ldots, s_{k^{\prime}}^{\prime} \in S$. 
Therefore, $\rho: \sum_{j=1}^{k} \delta\left(h_{j}, s_{j}\right) \mapsto \sum_{j=1}^{k} s_{j}$ defines a mapping of $D$ into $S$ which obviously is an additive homomorphism. We claim:

$$
K=\operatorname{ker} \rho \text {. }
$$

By (16) and the definition of $K$, we have $K \subseteq \operatorname{ker} \rho$. One generally has $\delta\left(h_{j}, s_{j}\right) \equiv \delta\left(h_{1}+s_{1}+\cdots+s_{j-1}, s_{j}\right) \bmod K$ for $h_{1}, \ldots, h_{j} \in H$, $s_{1}, \ldots, s_{j} \in S$. If now $\sum_{j=1}^{k} \delta\left(h_{j}, s_{j}\right) \in \operatorname{ker} \rho$, then $\sum_{j=1}^{k} s_{j}=0$, and consequently

$$
\begin{aligned}
\sum_{j=1}^{k} \delta\left(h_{j}, s_{j}\right) \equiv & \sum_{j=1}^{k} \delta\left(h_{1}+s_{1}+\cdots+s_{j-1}, s_{j}\right) \quad \bmod K \\
= & \left(\left(h_{1}+s_{1}\right) \hat{-} h_{1}\right) \hat{+}\left(\left(h_{1}+s_{1}+s_{2}\right) \hat{-}\left(h_{1}+s_{1}\right)\right) \\
& \hat{+} \cdots \hat{+}\left(\left(h_{1}+s_{1}+\cdots+s_{k}\right) \hat{-}\left(h_{1}+s_{1}+\cdots+s_{k-1}\right)\right) \\
= & \hat{-} h_{1} \hat{+}\left(h_{1}+s_{1}+\cdots+s_{k}\right)=0
\end{aligned}
$$

i.e., $\sum_{j=1}^{k} \delta\left(h_{j}, s_{j}\right) \in K$.

If $h \in H$ and $s \in S \backslash\{0\}$, then $\delta(h, s) \notin K$ by (19), and this means

$$
\text { is injective. }
$$

We now want to show that ${ }^{\sim}$ is injective which we shall conclude from

$$
h \wedge h^{\prime} \in D \Rightarrow h=h^{\prime}+s \quad \text { with } s \in S \text {, for all } h, h^{\prime} \in H \text {. }
$$

We reformulate (21) in the following form to make it accessible to an induction argument:

Suppose $h, h^{\prime} \in H$ and $r \in \mathbb{N}$. If there are $h_{1}, \ldots, h_{r} \in H$, (22) $s_{1}, \ldots, s_{r} \in S$ such that $h \hat{-} h^{\prime}=\sum_{j=1}^{r} \delta\left(h_{j}, s_{j}\right)$, then there is an element $s \in S$ such that $h=h^{\prime}+s$.

If $r=1$, then $h=h_{1}+s_{1}=h^{\prime}+s_{1}$, as $(T, \hat{+})$ is free over $H$. Now suppose $r>1$ and (22) is true for $r-1$ instead of $r$. Since $h \wedge h^{\prime}=$ $\sum_{j=1}^{r} \delta\left(h_{j}, s_{j}\right)$, we may assume $h=h_{1}+s_{1}, h^{\prime}=h_{r}$. Furthermore, $h_{1}=h_{i}$ $+s_{i}$ with $i \in\{2, \ldots, r\}$. This yields

$$
\begin{aligned}
h \hat{\sim} h^{\prime} & =\left(h_{i}+s_{i}+s_{1}\right) \hat{\sim}\left(h_{i}+s_{i}\right) \hat{+} \sum_{j=2}^{r} \delta\left(h_{j}, s_{j}\right) \\
& =\left(h_{i}+s_{i}+s_{1}\right) \hat{\sim} h_{i} \hat{+} \sum_{\substack{j=2 \\
j \neq i}}^{r} \delta\left(h_{j}, s_{j}\right) \\
& =\delta\left(h_{i}, s_{i}+s_{1}\right) \hat{+} \sum_{\substack{j=2 \\
j \neq i}}^{r} \delta\left(h_{j}, s_{j}\right),
\end{aligned}
$$


and an application of the induction hypothesis yields our claim. This proves (22) and the equivalent assertion (21) by means of which we conclude

$$
\sim \text { is injective: }
$$

For if $h, h^{\prime} \in H$ and $h \hat{\sim} h^{\prime} \in K$, then a fortiori $h \hat{\sim} h^{\prime} \in D$ and therefore $h=h^{\prime}+s$ with $s \in S$. But then $h \hat{\sim} h^{\prime}=\delta\left(h^{\prime}, s\right)$, and (20) implies $s=0$. Therefore we have $h=h^{\prime}$, and the proof of (23) is complete.

It remains to show that the envelopment $(R, \sim, \bar{\prime})$ is universal. To this end let $\left(R^{\prime}, \stackrel{=}{=}\right)$ be an envelopment of $\mathscr{F}$. Since $(T, \hat{+})$ is free over $H$,

$$
\chi_{0}: T \rightarrow R^{\prime}, \quad \sum_{j} z_{j} h_{j} \mapsto \sum_{j} z_{j} \tilde{h}_{j} \quad\left(z_{j} \in \mathbb{Z}\right)
$$

defines a ring homomorphism. We show

$$
K \subseteq \operatorname{ker} \chi_{0} \text {. }
$$

Let $h_{1}, \ldots, h_{k} \in H, s_{1}, \ldots, s_{k} \in S$ and $\sum_{j=1}^{k} \delta\left(h_{j}, s_{j}\right) \in K$. Then

$$
\begin{aligned}
\left(\sum_{j=1}^{k} \delta\left(h_{j}, s_{j}\right)\right)^{\chi_{0}} & =\sum_{j=1}^{k}\left(\left(h_{j}+s_{j}\right)^{x_{0}}-h_{j}^{\chi_{0}}\right) \\
& =\sum_{j=1}^{k}\left(\widetilde{\widetilde{h_{j}+s_{j}}}-\tilde{h}_{j}\right)=\sum_{j=1}^{k}\left(\tilde{\tilde{h}}_{j}+\overline{\bar{s}}_{j}-\tilde{h}_{j}\right) \\
& =\sum_{j=1}^{k} \overline{\overline{\bar{s}_{j}}}=\overline{\sum_{j=1}^{k} s_{j}}=0,
\end{aligned}
$$

by (9) and (19).

By (24),

$$
\chi: R \rightarrow R^{\prime}, \quad \sum_{j} z_{j} h_{j} \hat{+} K \mapsto \sum_{j} z_{j} \tilde{h}_{j} \quad\left(z_{j} \in \mathbb{Z}\right)
$$

defines a homomorphism, and for all $h \in H, s \in S$ we have

$$
\begin{aligned}
& \tilde{h}^{x}=(h \hat{+} K)^{x}=\tilde{h}, \\
& \bar{s}^{x}=(\delta(h, s) \hat{+} K)^{x}=\widetilde{\tilde{h+s}}-\tilde{h}=\tilde{h}+\overline{\bar{s}}-\tilde{h}=\overline{\bar{s}},
\end{aligned}
$$

by (9). This completes the proof of our theorem.

\section{Fitting pre-structures.}

2.1. Definition. Let $H$ be a semigroup, $S$ a ring, $\varphi$ a homomorphism of $H$ into the multiplicative semigroup of $\operatorname{End}_{\Lambda}(S), \psi$ an antihomomorphism of $H$ into the multiplicative semigroup of $\operatorname{End}_{\mathrm{P}}(S)$ such 
that $H^{\varphi}$ and $H^{\psi}$ commute elementwise. (We use the notations introduced in 1.1.) The 4-tuple $\mathscr{F}_{0}:=(H, S, \varphi, \psi)$ is called a Fitting pre-structure if condition (1) holds.

Let $\mathscr{F}=\left(H^{*}, S^{*}, \varphi^{*}, \psi^{*}, \sigma^{*}\right)$ be a Fitting structure with $S=S^{*}$, and $\mu$ a monomorphism of $H$ into $H^{*}$. The pair $(\mathscr{F}, \mu)$ is called a continuation of $\mathscr{F}_{0}$ if

$$
\varphi=\mu \varphi^{*}, \quad \psi=\mu \psi^{*}
$$

holds.

Isomorphisms of Fitting pre-structures and Fitting sub-pre-structures are defined in complete analogy to the corresponding notions for Fitting structures, the conditions on $\sigma, \sigma^{\prime}$ being omitted.

If $\left(\mathscr{F}_{1}, \mu_{1}\right)$ and $\left(\mathscr{F}_{2}, \mu_{2}\right)$ with $\mathscr{F}_{1}=\left(H^{*}, S^{*}, \varphi^{*}, \psi^{*}, \sigma^{*}\right), \quad \mathscr{F}_{2}=$ $\left(H^{* *}, S^{* *}, \varphi^{* *}, \psi^{* *}, \sigma^{* *}\right)$ are continuations of $\mathscr{F}_{0}$, then a homomorphism of $\left(\mathscr{F}_{1}, \mu_{1}\right)$ into $\left(\mathscr{F}_{2}, \mu_{2}\right)$ is defined to be a homomorphism $\omega$ of $H^{*}$ into $H^{* *}$ with the property

$$
\mu_{2}=\mu_{1} \omega \text {. }
$$

If $\omega$ is a bijection of $H^{*}$ onto $H^{* *}$, our continuations are called isomorphic. A continuation $\mathscr{F}$ of $\mathscr{F}_{0}$ is called universal if for any continuation $\mathscr{F}^{\prime}$ of $\mathscr{F}_{0}$ there is a homomorphism of $\mathscr{F}$ into $\mathscr{F}^{\prime}$.

Universal continuations of isomorphic Fitting pre-structures are isomorphic. We now prove the following existence theorem:

\subsection{THEOREM. Every Fitting pre-structure has a universal continuation.}

Proof. Let $\mathscr{F}_{0}=(H, S, \varphi, \psi)$ be the Fitting pre-structure given. We put $H^{\mathscr{F}_{0}}:=H \times S$ and define

$$
\left(h_{1}, s_{1}\right)\left(h_{2}, s_{2}\right):=\left(h_{1} h_{2}, s_{1} h_{2}+h_{1} s_{2}+s_{1} s_{2}\right)
$$

for all $h_{1}, h_{2} \in H, s_{1}, s_{2} \in S$. One readily verifies that $H^{\mathscr{F}_{0}}$ is a semigroup and the mapping

$$
\mu: H \rightarrow H^{\mathscr{\mathscr { T } _ { 0 }}}, \quad h \mapsto(h, 0)
$$

is a monomorphism. We call $H^{\mathscr{F}_{0}}$ the continuation semigroup of $\mathscr{F}_{0}$. We put $S^{*}:=S$, and define for all $h \in H, s \in S$

$$
\begin{array}{ll}
(h, s)^{\varphi^{*}}: S \rightarrow S, & r \mapsto r h+r s \\
(h, s)^{\psi^{*}}: S \rightarrow S, & r \mapsto h r+s r .
\end{array}
$$

Then $(h, s)^{\varphi^{*}} \in \operatorname{End}_{\Lambda}(S),(h, s)^{\psi^{*}} \in \operatorname{End}_{\mathrm{P}}(S)$, since

$$
\begin{aligned}
& \left(r_{1} r\right) h+\left(r_{1} r\right) s=r_{1}(r h)+r_{1}(r s)=r_{1}(r h+r s), \\
& h\left(r r_{1}\right)+s\left(r r_{1}\right)=(h r) r_{1}+(s r) r_{1}=(h r+s r) r_{1}
\end{aligned}
$$


for all $h \in H, r, r_{1}, s \in S$. We show

(27) $\varphi^{*}$ is a homomorphism of $H^{\mathscr{\mathscr { F } _ { 0 }}}$ into $\operatorname{End}_{\Lambda}(S), \psi^{*}$ is an antihomomorphism of $H^{\mathscr{F _ { 0 }}}$ into $\operatorname{End}_{\mathrm{P}}(S)$, and (25) holds.

We confine ourselves to the assertions about $\varphi, \varphi^{*}$ and leave the proof of the assertions about $\psi, \psi^{*}$ to the reader. For all $h, h_{1}, h_{2} \in H, r, s_{1}, s_{2} \in S$ we have

$$
\begin{aligned}
r^{\left(\left(h_{1}, s_{1}\right)\left(h_{2}, s_{2}\right)\right)^{\varphi^{*}}} & =r^{\left(h_{1} h_{2}, s_{1} h_{2}+h_{1} s_{2}+s_{1} s_{2}\right)^{\varphi^{*}}} \\
& =\left(r h_{1}+r s_{1}\right) h_{2}+\left(r h_{1}+r s_{1}\right) s_{2}=r^{\left(h_{1}, s_{1}\right)^{\varphi^{*}}\left(h_{2}, s_{2}\right)^{\varphi^{*}}}
\end{aligned}
$$

and

$$
r^{h^{\varphi}}=r h+r \cdot 0=r^{(h, 0)^{\varphi^{*}}}=r^{h^{\mu \varphi^{*}}} .
$$

In the following we write (as in 1.1) $r(h, s)$ for $r^{(h, s)^{\phi^{*}}},(h, s) r$ for $r^{(h, s)^{\psi^{*}}}$ and verify

$$
H^{\varphi^{*}} \text { and } H^{\psi^{*}} \text { commute elementwise, }
$$

as for all $h_{1}, h_{2} \in H, r, s_{1}, s_{2} \in S$

$$
\begin{aligned}
\left(\left(h_{1}, s_{1}\right) r\right)\left(h_{2}, s_{2}\right) & =\left(h_{1} r+s_{1} r\right)\left(h_{2}, s_{2}\right) \\
& =\left(h_{1} r+s_{1} r\right) h_{2}+\left(h_{1} r+s_{1} r\right) s_{2} \\
& =h_{1}\left(r h_{2}+r s_{2}\right)+s_{1}\left(r h_{2}+r s_{2}\right) \\
& =\left(h_{1}, s_{1}\right)\left(r h_{2}+r s_{2}\right)=\left(h_{1}, s_{1}\right)\left(r\left(h_{2}, s_{2}\right)\right) .
\end{aligned}
$$

A similar standard calculation yields

$$
\left(r_{1}(h, s)\right) r_{2}=r_{1}\left((h, s) r_{2}\right) \quad \text { for all } h \in H, r_{1}, r_{2}, s \in S,
$$

i.e., condition (1) is satisfied.

For all $r \in S$ we put

$$
r^{\sigma^{*}}: H^{\mathscr{F}_{0}} \rightarrow H^{\mathscr{F}_{0}}, \quad(h, s) \mapsto(h, r+s) .
$$

Then $r^{\sigma^{*}}$ is a permutation of $H^{\mathscr{F}_{0}}$. As before, we write $(h, s)+r$ for $(h, s)^{r^{\sigma^{*}}}$ and observe

$$
\sigma^{*} \text { is a homomorphism of }(S,+) \text { into } \widetilde{S}_{H^{\Phi_{0}}}
$$

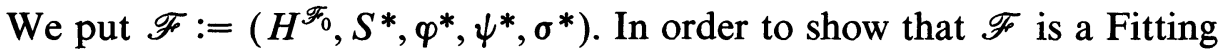
structure it remains to check conditions (2) and (3) which here turn into

$$
\begin{aligned}
& \left(\left(h_{1}, s_{1}\right)+r\right)\left(h_{2}, s_{2}\right)=\left(h_{1}, s_{1}\right)\left(h_{2}, s_{2}\right)+r\left(h_{2}, s_{2}\right), \\
& \left(h_{1}, s_{1}\right)\left(\left(h_{2}, s_{2}\right)+r\right)=\left(h_{1}, s_{1}\right)\left(h_{2}, s_{2}\right)+\left(h_{1}, s_{1}\right) r
\end{aligned}
$$

for all $h_{1}, h_{2} \in H, r, s_{1}, s_{2} \in S$, 


$$
\begin{aligned}
& \left((h, s)+r_{1}\right) r_{2}=(h, s) r_{2}+r_{1} r_{2}, \\
& r_{1}\left((h, s)+r_{2}\right)=r_{1}(h, s)+r_{1} r_{2},
\end{aligned}
$$

for all $h \in H, s, r_{1}, r_{2} \in S$, both being immediate consequences of our definitions. Now (27) and (30) show that $(\mathscr{F}, \mu)$ is a continuation of $\mathscr{F}_{0}$, and we claim:

$$
\mathscr{F} \text { is universal. }
$$

For if $\left(\mathscr{F}^{\prime}, \mu^{\prime}\right)$ with $\mathscr{F}^{\prime}=\left(H^{* *}, S^{* *}, \varphi^{* *}, \psi^{* *}, \sigma^{* *}\right)$ is a continuation of $\mathscr{F}_{0}$, we put

$$
\omega: H^{\mathscr{\mathscr { F } _ { 0 }}} \rightarrow H^{* *}, \quad(h, s) \mapsto h^{\mu^{\prime}}+s
$$

and calculate for $h_{1}, h_{2} \in H, s_{1}, s_{2} \in S$ by means of (25):

$$
\begin{aligned}
\left(\left(h_{1}, s_{1}\right)\left(h_{2}, s_{2}\right)\right)^{\omega} & =\left(h_{1} h_{2}, s_{2}^{h^{\psi^{*}}}+s_{1}^{h_{2}^{\mathscr{S}^{*}}}+s_{1} s_{2}\right)^{\omega} \\
& =\left(h_{1} h_{2}\right)^{\mu^{\prime}}+s_{2}^{h^{\psi^{*}}}+s_{1}^{h^{*}}+s_{1} s_{2} \\
& =h_{1}^{\mu^{\prime}} h_{2}^{\mu^{\prime}}+s_{2}^{h_{1}^{\mu^{\prime} \psi^{* *}}}+s_{1}^{h_{2}^{\mu^{\prime} \varphi^{* *}}}+s_{1} s_{2} \\
& =\left(h_{1}^{\mu^{\prime}}+s_{1}\right)\left(h_{2}^{\mu^{\prime}}+s_{2}\right)=\left(h_{1}, s_{1}\right)^{\omega}\left(h_{2}, s_{2}\right)^{\omega},
\end{aligned}
$$

whence $\omega$ is a homomorphism. Since for all $h \in H$ we have $h^{\mu \omega}=(h, 0)^{\omega}$ $=h^{\mu^{\prime}}$, we put $\mu^{\prime}=\mu \omega$ so that (26) holds. Thus the proof of our theorem is complete.

We add some remarks on the continuation semigroup $H^{\mathscr{F}_{0}}$ of a Fitting pre-structure $\mathscr{F}_{0}$. For any ring $S$,

$$
s_{1} \circ s_{2}:=s_{1}+s_{2}+s_{1} s_{2} \quad\left(s_{1}, s_{2} \in S\right)
$$

defines an associative composition with identity element 0 . As is well known, $S$ is a radical ring if and only if $(S, \circ)$ is a group. We have:

If $H$ has an identity element 1 , then

$$
\nu: S \rightarrow H^{\mathscr{F}_{0}}, s \mapsto(1, s)
$$

is a monomorphism of $(S, \circ)$ into $H^{\mathscr{F}_{0}}$.

(36) If $H$ has a zero element 0 , then

$$
\lambda: S \rightarrow H^{\mathscr{F _ { 0 }}}, s \mapsto(0, s)
$$

is a monomorphism of $(S, \cdot)$ into $H^{\mathscr{F}_{0}}$.

An element $\left(h_{0}, s_{0}\right) \in H^{\mathscr{F}_{0}}$ is an identity element of $H^{\mathscr{F _ { 0 }}}$ if and only if $h_{0}$ is an identity element of $H$, $s_{0} H=0=H s_{0}$ and $\left(h_{0}+s_{0}\right)^{\varphi}=\mathrm{id}_{S}=\left(h_{0}+s_{0}\right)^{\psi}$,

since $\left(h_{0}, s_{0}\right)$ is an identity element of $H^{\mathscr{F _ { 0 }}}$ if and only if $h_{0} h=h=h h_{0}$ and $h_{0} s+s_{0} h+s_{0} s=s=h s_{0}+s h_{0}+s_{0} s$ for all $h \in H, s \in S$. 
We obviously have

(38) If $H^{\mathscr{F}_{0}}$ is a group, then so is $H$.

(39) If $H$ has an identity element 1 such that $1^{\varphi}=\mathrm{id}_{S}=1^{\psi}$, then $(1,0)$ is an identity element of $H^{\mathscr{\mathscr { F } _ { 0 }}}$, and $H^{\mathscr{F}_{0}}$ is a group if and only if $H$ is a group and $S$ is a radical ring. In this case $H^{\mathscr{F}_{0}}$ is a semdirect product of $H$ and $(S, \circ)$.

Herein the statement about $(1,0)$ follows from (37). Now let $H$ be a group and $S$ a radical ring. If for $h \in H, s \in S$ the $\circ$-inverse element of $s h^{-1}$ is denoted by $\left(s h^{-1}\right)^{-}$, we have

$$
(h, s)\left(h^{-1}, h^{-1}\left(s h^{-1}\right)^{-}\right)=\left(1,1\left(s h^{-1}\right)^{-}+s h^{-1}+s h^{-1}\left(s h^{-1}\right)^{-}\right)=(1,0) .
$$

Therefore $H^{\mathscr{F _ { 0 }}}$ is a group. As to the converse, observing (38), it suffices to show that $S$ is a radical ring. But if $s \in S$ and $\left(h_{1}, s_{1}\right) \in H^{\mathscr{F}_{0}}$ is the inverse of $(1, s)$, then

$$
\begin{aligned}
& (1,0)=(1, s)\left(h_{1}, s_{1}\right)=\left(h_{1}, 1 \cdot s_{1}+s h_{1}+s s_{1}\right), \\
& (1,0)=\left(h_{1}, s_{1}\right)(1, s)=\left(h_{1}, h_{1} s+s_{1} \cdot 1+s_{1} s\right),
\end{aligned}
$$

hence $h_{1}=1$ and $s_{1} \circ s=0=s \circ s_{1}$. Thus $s$ is $\circ$-invertible. Let finally $\mu$ be the embedding of $H$ into $H^{\mathscr{F _ { 0 }}}$ as in the proof of 2.2 and $\nu$ as in (35). Then $S^{\nu}$ is a normal subgroup of $H^{\mathscr{\mathscr { T } _ { 0 }}}$ and isomorphic to $(S, \circ), H^{\mu}$ is a subgroup of $H^{\mathscr{F _ { 0 }}}$ which is isomorphic to $H$ such that $S^{\nu} \cap H^{\nu}=\{(1,0)\}$, and for all $h \in H, s \in S$ we have

$$
(h, s)=\left(1, s h^{-1}\right)(h, 0)=\left(s h^{-1}\right)^{\nu} h^{\mu},
$$

whence $H^{\mathscr{F}_{0}}=S^{\nu} H^{\mu}$.

In order to give examples of Fitting structures, it is sufficient, by 2.2 , to construct Fitting pre-structures:

2.3. ExAmple. Let $M$ be a set and $A$ a subset of $M$ which is an abelian group with respect to some composition + . Then the set

$$
S(M, A):=\left\{s|s: M \rightarrow A, s|_{A} \in \operatorname{End}(A)\right\}
$$

is a ring with respect to the compositions

$$
\begin{aligned}
s_{1}+s_{2}: & M \rightarrow A \\
s_{1} s_{2}: & M \mapsto m^{s_{1}}+m^{s_{2}} \\
& M \mapsto A \\
& m \mapsto r\left(m^{s_{1}}\right)^{s_{2}},
\end{aligned}
$$


and the set

$$
H(M, A):=\left\{h|h: M \rightarrow M, h|_{A} \in \operatorname{End}(A)\right\}
$$

is a semigroup with respect to the composition

$$
\begin{aligned}
h_{1} h_{2}: & M \rightarrow M \\
& m \mapsto\left(m^{h_{1}}\right)^{h_{2}} .
\end{aligned}
$$

For all $h \in H(M, A), s \in S(M, A)$ let $s^{h^{\Phi}}$ (resp. $s^{h^{\psi}}$ ) be the usual composition (of mappings) $s h$ (resp. $h s$ ).

Then $(H(M, A), S(M, A), \Phi, \Psi)$ is a Fitting pre-structure.

We show that all "well-behaved" Fitting pre-structures can be subsumed under this type of example:

2.4. TheOREM. Let $H$ be a semigroup with identity element $1, \mathscr{F}=$ $(H, S, \varphi, \psi)$ a Fitting pre-structure such that $1^{\psi}=\mathrm{id}_{s}$. Then there are $M, A$ as in 2.3 such that $\mathscr{F}$ is isomorphic to a Fitting sub-pre-structure of $(H(M, A), S(M, A), \Phi, \Psi)$.

Proof. W.1.o.g. we may assume $H \cap S=\varnothing$. Then we put $M:=H \cup S,(A,+):=(S,+)$. For all $h \in H, s \in S$ we define

$$
\begin{array}{ll}
h^{\alpha}: M \rightarrow M, & m \mapsto m h= \begin{cases}m h & \text { for } m \in H \\
m^{h^{\varphi}} & \text { for } m \in S\end{cases} \\
s^{\beta}: M \rightarrow A, & m \mapsto m s= \begin{cases}s^{m^{\psi}} & \text { for } m \in H \\
m s & \text { for } m \in S .\end{cases}
\end{array}
$$

Obviously, $\alpha$ is a homomorphism of $H$ into $H(M, A)$, and $\beta$ is a homomorphism of $S$ into $S(M, A)$. For $h \in \operatorname{ker} \alpha$ we have $1=1^{h^{\alpha}}=$ $1 \cdot h=h$; thus $\alpha$ is injective. Similarly, $s \in \operatorname{ker} \beta$ implies $0=1^{s^{\beta}}=1 \cdot s$ $=s$, hence $\beta$ is injective, too. For all $m \in M, h \in H, s \in S$ we have

$$
\begin{aligned}
& m^{h^{\alpha} s^{\beta}}=(m h) s=m(h s)=m^{(h s)^{\beta}}, \\
& m^{s^{\beta} h^{\alpha}}=(m s) h=m(s h)=m^{(s h)^{\beta}}, \\
& m^{\left(s^{\beta}\right)^{\left(h^{\alpha}\right)^{\Phi}}}=m^{s^{\beta} h^{\alpha}}=(m s) h=m(s h) \\
& =m^{(s h)^{\beta}}=m^{s^{\left(h \phi_{\beta}\right)}}=m^{\left(s^{\beta}\right)^{\beta^{-1} h_{\beta}}}=m^{\left(s^{\beta}\right)^{(h \varphi) \bar{\beta}}}, \\
& m^{\left(s^{\beta}\right)^{(h \alpha) \Psi}}=m^{h^{\alpha} s^{\beta}}=(m h) s=m(h s) \\
& =m^{(h s)^{\beta}}=m^{s^{\left(h^{\psi} \beta\right)}}=m^{\left(s^{\beta}\right)^{\beta^{-1} h \beta}}=m^{\left(s^{\beta}\right)^{(h \psi /)}} .
\end{aligned}
$$


Hence $H^{\alpha} S^{\beta} \subseteq S^{\beta}, \quad S^{\beta} H^{\alpha} \subseteq S^{\beta}, \quad \alpha \Phi=\varphi \bar{\beta}, \quad \alpha \Psi=\psi \bar{\beta}$. Therefore $\left(H^{\alpha}, S^{\beta},\left.\Phi\right|_{H^{\alpha}},\left.\Psi\right|_{H^{\alpha}}\right)$ is a Fitting sub-pre-structure of $(H(M, A), S(M, A)$, $\Phi, \Psi)$ and isomorphic to $(H, S, \varphi, \psi)$. The hypothesis that $H$ has an identity element 1 such that $1^{\psi}=\mathrm{id}_{S}$ has only been used to prove that $\alpha$ and $\beta$ are injective. As is easily seen, for that purpose even weaker hypotheses on $H$ are sufficient.

Let $G$ be a group and $A$ a characteristic abelian normal subgroup of $G$. Then $\mathscr{F}:=(H(G, A), S(G, A), \Phi, \Psi)$ is a Fitting pre-structure. If we put $H:=\operatorname{Aut}(G), S:=\operatorname{Hom}(G, A)$, then we obviously have $H S \subseteq S$, $S H \subseteq S$, whence $(H, S, \varphi, \psi)$ with $\varphi=\left.\Phi\right|_{H}, \psi=\left.\Psi\right|_{H}$ is a Fitting subpre-structure of $\mathscr{F}$. If we put for $h \in H(G, A), s \in S(G, A)$

$$
h^{s^{\Sigma}}: G \rightarrow G, \quad g \mapsto g^{h} g^{s},
$$

then $s^{\Sigma} \in \mathbb{S}_{H(G, A)}$, and $\Sigma$ is a homomorphism of $(S(G, A),+)$ into $\Im_{H(G, A)}$ such that $h^{s^{\Sigma}}=h \Leftrightarrow s=0$ for all $h \in H(G, A), s \in S(G, A)$. It is easy to see that (2) and (3) hold; thus ( $H(G, A), S(G, A), \Phi, \Psi, \Sigma)$ is a Fitting structure. We put $\sigma:=\left.\Sigma\right|_{H}$. In general, $(H, S, \varphi, \psi, \sigma)$ need not be a Fitting structure. But we have:

2.5. THEOREM. Let $G$ be a group which has no nontrivial direct abelian factor. Assume $Z(G)$ is finite. Then $(\operatorname{Aut}(G), \operatorname{Hom}(G, Z(G)), \varphi, \psi, \sigma)$ is a Fitting structure.

( Here $\varphi, \psi, \sigma$ have the meaning introduced above.)

Proof. By our preparatory considerations it suffices to show:

(40) $\alpha^{\zeta^{\sigma}} \in \operatorname{Aut}(G)$ for all $\alpha \in \operatorname{Aut}(G), \zeta \in \operatorname{Hom}(G, Z(G))$.

Since $\alpha^{\zeta^{\sigma}} \in \operatorname{Aut}(G)$ if and only if $\operatorname{id}_{G}^{\left(\alpha^{-1} \zeta\right)^{\sigma}} \in \operatorname{Aut}(G)$, for our proof of (40) we may assume $\alpha=\mathrm{id}_{G}$. Surely, $\mathrm{id}_{G}^{\zeta^{\sigma}}$ is a homomorphism. By our hypotheses on $G$ and $Z(G)$, we have (see [1]) $\zeta^{n}=0$ for an appropriate $n \in \mathbb{N}$. Since

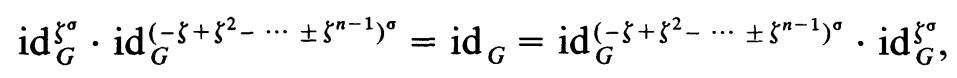

$\mathrm{id}_{G}^{\zeta^{o}}$ is bijective, proving (40).

\section{Wreath products over Fitting structures.}

3.1. Definition. Let $\mathscr{F}=(H, S, \varphi, \psi, \sigma)$ be a Fitting structure and $n \in \mathbb{N}$. For $\pi \in \mathbb{S}_{n}$, a $(n \times n)$-matrix $A=\left(a_{i j}\right)$ is called a $\pi$-matrix over $H \cup S$ if

$$
a_{i j} \in \begin{cases}H & \text { for } j=i \pi \\ S & \text { for } j \neq i \pi\end{cases}
$$


If $\pi, \pi^{\prime} \in \mathbb{S}_{n}$ and $\left(a_{i j}\right)$ is a $\pi$-matrix, $\left(a_{i j}^{\prime}\right)$ is a $\pi^{\prime}$-matrix over $H \cup S$, we define, using the product and sum notations introduced in 1.1,

$$
\left(a_{i j}\right)\left(a_{i j}^{\prime}\right):=\left(b_{i j}\right) \quad \text { with } b_{i j}=\sum_{k=1}^{n} a_{i k} a_{k j}^{\prime} \quad \text { for } 1 \leq i, j \leq n .
$$

We observe:

(41) If $\pi, \pi^{\prime} \in \mathfrak{S}_{n}$ and $A$ is a $\pi$-matrix, $A^{\prime}$ is a $\pi^{\prime}$-matrix over $H \cup S$, then $A A^{\prime}$ is a $\left(\pi \pi^{\prime}\right)$-matrix over $H \cup S$.

3.2. Definition. Let $\mathscr{F}=(H, S, \varphi, \psi, \sigma)$ be a Fitting structure and $n \in \mathbb{N}$. Let $X$ be a subgroup of $\mathfrak{S}_{n}$. We put

$$
H \underset{S}{\gamma_{S}} X:=\{(A, \pi) \mid \pi \in X, A \text { is a } \pi \text {-matrix over } H \cup S\},
$$

and define for $(A, \pi),\left(A^{\prime}, \pi^{\prime}\right) \in H \backslash_{S} X$

$$
(A, \pi)\left(A^{\prime}, \pi^{\prime}\right):=\left(A A^{\prime}, \pi \pi^{\prime}\right) .
$$

By (41), this is a composition in $H \Upsilon_{S} X$. We observe:

$$
H \underset{S}{\chi_{S}} X \text { is a semigroup. }
$$

We call $H \backslash X$ the wreath product of $H$ and $X$ over $S$. If $H \cap S=\varnothing$

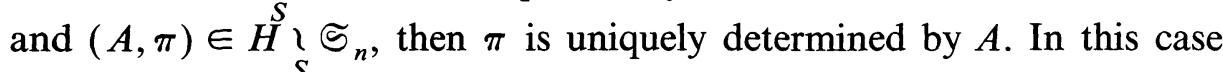
the elements $(A, \stackrel{S}{\pi})$ of the wreath product can be identified with their first components, the matrices $A$.

We add a few simple remarks:

(43) If $H=S$ and $X$ is the trivial subgroup of $\mathfrak{S}_{n}$, then $H \backslash X$ is isomorphic to the multiplicative semigroup of the ring $(S)_{n}$ of all $(n \times n)$-matrices over $S$.

(44) If $\mathscr{F}^{\prime}=\left(H^{\prime}, S^{\prime}, \varphi^{\prime}, \psi^{\prime}, \sigma^{\prime}\right)$ is a Fitting substructure of $\mathscr{F}$ and $X^{\prime}$ is a subgroup of $X$, then $H^{\prime} \backslash X^{\prime}$ is a subsemigroup of $H>_{S} X$.

(45) The standard wreath product $H \backslash X$ is isomorphic to $H$ < $X$ (writing $S_{0}$ for the trivial ring); thus it is contained $S_{0}$ in every wreath product $H \Upsilon_{S} X$ as a subsemigroup.

For $\left(H,\{0\}, \varphi_{0}, \psi_{0},\left.\sigma\right|_{\{0\}}\right)$ is a Fitting substructure of $(H, S, \varphi, \psi, \sigma)$ where we write $\varphi_{0}, \psi_{0}$ for the (unique) actions of $H$ on $\{0\}$, whence the second part of (45) is a consequence of (44). 
Matrix multiplications yield actions of $H_{\{0\}} X$ on $(S)_{n}$ : We put $B^{A^{\hat{\Phi}}}:=B A, \quad B^{A^{\hat{\psi}}}=A B$ for $(A, \pi) \stackrel{\{0\}}{\in} H_{\{0\}} X, B \in(S)_{n}$,

where these products are defined analogously to the matrix product introduced above. Then one readily verifies that $\mathscr{F}_{0}:=\left(H \backslash X,(S)_{n}\right.$, $\hat{\varphi}, \hat{\psi})$ is a Fitting pre-structure. The mapping $\kappa$ of the continuation semigroup $(H \backslash X)^{\mathscr{F}_{0}}$ into $H \backslash X$ such that $(A, B)^{\kappa}=A+B$ for all

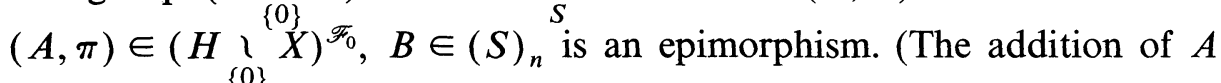
and $B$ means, as usual, addition of corresponding components, using the notations of 1.1 with regard to $\sigma$.) If $H$ has an identity element 1 , then

$$
\operatorname{ker} \kappa=\left\{\left(\begin{array}{ccc}
1+s_{1} & & 0 \\
& \ddots & \\
0 & & 1+s_{n}
\end{array}\right) \mid s_{j} \in S\right\} \cong(S \oplus \underset{n}{\ldots} \oplus S, \circ) \text {. }
$$

We claim

(46) Let $\mathscr{F}=(H, S, \varphi, \psi, \sigma)$ be a Fitting structure, $n \in \mathbb{N}$ and $X$ a subgroup of $\mathfrak{S}_{n}$. Suppose $H$ has an identity element 1. Then $H \backslash_{S} X$ is a group if and only if $H$ is a group and $S$ is a radical ring.

For, if $H$ is a group and $S$ is a radical ring, then, by (45), $H$ \ $X$ is a group and, by [3, I, 7. Th. 3], $(S)_{n}$ is a radical ring. Therefore, (39) yields

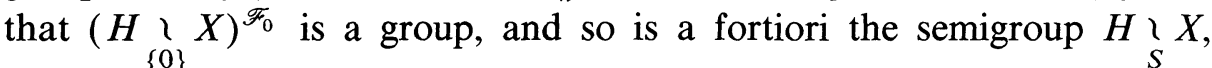
being isomorphic to $(H \backslash X)^{\mathscr{F}_{0}} / \operatorname{ker} \kappa$. Conversely, suppose $H \backslash X$ is a group. Its identity element being denoted by $(I, \mathrm{id})$, where $I$ is the identity matrix, we have $(I+B$, id $) \in H \backslash X$ for every $B \in(S)_{n}$. If we put $C:=(I+B)^{-1}$, then $B C \in(S)_{n}$, and

$$
\begin{aligned}
& B \circ(-B C)=B-B C-B^{2} C=B-B(I+B) C=B-B I=0, \\
& (-B C) \circ B=-B C+B-B C B=B-B C(I+B)=B-B I=0 .
\end{aligned}
$$

Therefore $(S)_{n}$, hence $S$, is a radical ring. Now let $h \in H$ and set

$$
A:=\left(\begin{array}{llll}
h & & & 0 \\
& 1 & & \\
0 & & & 1
\end{array}\right) .
$$

As before, we have $(A, \mathrm{id}) \in H \backslash X$, and the entry $c$ in the upper left corner of $A^{-1}$ satisfies $h c=1=\stackrel{S}{ }$. The assumption $c \in S$ would imply $1 \in S$ and, regarding the equations $1^{\varphi}=\mathrm{id}_{S}=1^{\psi}, 1$ were an identity 
element of $S$. As $S$ is a radical ring, this would yield $S=\{1\} \subseteq\{1\} \cdot H$ $\subseteq S$, whence $S=\{1\}=H$, and everything would be trivial. But if $c \notin S$, then $c \in H$, and $h$ is an invertible element of $H$, as desired.

We finally show that our notion of a generalized wreath product is useful for the description of automorphism groups of groups:

To this end let $G$ be a direct indecomposable nonabelian group satisfying the minimum condition on central subgroups, and let $n \in \mathbb{N}$. We put $S:=\operatorname{Hom}(G, Z(G))$. Then $S$ is a nil ring, hence a radical ring. By 2.5, $(\operatorname{Aut}(G), S, \varphi, \psi, \sigma)$ is a Fitting structure, and the associated wreath product $(\operatorname{Aut}(G)) \backslash \Im_{n}$ is a group, by (46). Since $\operatorname{Aut}(G) \cap S=\varnothing$, we may identify its elements $(A, \pi)$ with their first components, the matrices $A$. For $A=\left(\alpha_{i j}\right) \in(\operatorname{Aut}(G)) \underset{S}{\backslash \subseteq_{n}}$ we define

$$
\left(g_{1}, \ldots, g_{n}\right) A:=\left(\prod_{i=1}^{n} g_{i}^{\alpha_{i 1}}, \ldots, \prod_{i=1}^{n} g_{i}^{\alpha_{i n}}\right) \quad \text { for } g_{1}, \ldots, g_{n} \in G .
$$

This gives a mapping $\alpha_{A}$ of $G \times \cdots \times G$ ( $n$ factors) into itself which can formally be regarded as the multiplication of the row $\left(g_{1}, \ldots, g_{n}\right)$ and the matrix $A$. The properties of $A$ imply:

For $g_{1}, \ldots, g_{n} \in G, j \in\{1, \ldots, n\}$, there is at most one

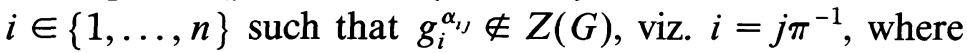
$\pi$ is the permutation determined by $A$.

Therefore for $g_{1}, \ldots, g_{n}, h_{1}, \ldots, h_{n} \in G, j \in\{1, \ldots, n\}$ we have

$$
\prod_{i=1}^{n}\left(g_{i} h_{i}\right)^{\alpha_{i \jmath}}=\prod_{i=1}^{n} g_{i}^{\alpha_{\imath}} h_{i}^{\alpha_{i \jmath}}=\prod_{i=1}^{n} g_{i}^{\alpha_{i j}} \prod_{i=1}^{n} h_{i}^{\alpha_{\imath \jmath}}
$$

yielding

For all $A \in(\operatorname{Aut}(G)) \underset{S}{\Im_{n}}, \alpha_{A}$ is an endomorphism of $G \times \underset{n}{\cdots} \times G$.

For $A=\left(\alpha_{i j}\right), B=\left(\beta_{i j}\right) \in(\operatorname{Aut}(G)) \chi_{S} \subseteq_{n}, g_{1}, \ldots, g_{n} \in G$ and $j \in$ $\{1, \ldots, n\}$, we have, by (48)

$$
\prod_{i=1}^{n} g_{i}^{\sum_{k=1}^{n} \alpha_{i k} \beta_{k j}}=\prod_{i=1}^{n}\left(\prod_{k=1}^{n} g_{i}^{\alpha_{t k}}\right)^{\beta_{k j}}=\prod_{k=1}^{n}\left(\prod_{i=1}^{n} g_{i}^{\alpha_{t k}}\right)^{\beta_{k j}}
$$

which implies

$$
\alpha_{A} \alpha_{B}=\alpha_{A B} \text { for all } A, B \in(\operatorname{Aut}(G)) \imath_{S} \subseteq_{n} .
$$

This and the obvious statement

$$
\alpha_{I}=\mathrm{id} \text {, where } I \text { is the identity element of }(\operatorname{Aut}(G))_{S} \Im_{n}
$$


imply:

(52) Associating to each $A \in(\operatorname{Aut}(G)) \chi_{S} \Im_{n}$ the automorphism $\alpha_{A}$ yields a homomorphism $\iota$ of $(\operatorname{Aut}(G)){ }_{S} \widetilde{S}_{n}$ into $\operatorname{Aut}(G \times \cdots \times G)$ ( $n$ factors).

If $A \in(\operatorname{Aut}(G)) \backslash_{S} \subseteq_{n}$ such that $\alpha_{A}=\mathrm{id}_{G \times \cdots \times G}$ ( $n$ factors), then for all $i \in\{1, \ldots, n\}, g \stackrel{S}{g} \in$

$$
(1, \ldots, 1, g, 1, \ldots, 1)=(1, \ldots, 1, g, 1, \ldots, 1) A=\left(g^{\alpha_{i 1}}, \ldots, g^{\alpha_{i n}}\right),
$$

where $g$ is in the $i$ th place. Hence $A=I$. Thus we have

$\iota$ is injective.

Finally we claim $\iota$ is surjective.

To this end we define for all $j \in\{1, \ldots, n\}$

$$
\begin{aligned}
& \varepsilon_{j}: \quad G \rightarrow G \times \underset{n}{\ldots} \times G \\
& g \mapsto(1, \ldots, 1, g, 1, \ldots, 1), \\
& \text { (where } g \text { is in the } j \text { th place) } \\
& \delta_{j}: \quad G \times \underset{n}{\ldots} \times G \rightarrow G \\
& \left(g_{1}, \ldots, g_{n}\right) \mapsto g_{j},
\end{aligned}
$$

and put for all $\alpha \in \operatorname{Aut}(G \times \cdots \times G)$

$$
A_{\alpha}:=\left(\alpha_{i j}\right) \quad \text { with } \alpha_{i j}^{n}=\varepsilon_{i} \alpha \delta_{j} \quad \text { for } i, j \in\{1, \ldots, n\} \text {. }
$$

Then $\alpha_{i j}$ is an endomorphism of $G$, and by [1, Satz 2] there is exactly one $\pi \in \mathfrak{S}_{n}$ such that, for $i \in\{1, \ldots, n\}, \alpha_{i, i \pi} \in \operatorname{Aut}(G)$ and $\alpha_{i j} \in S$ for

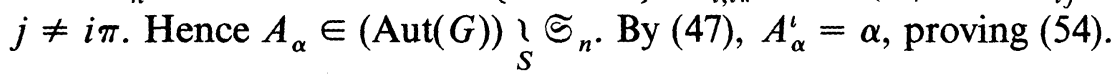

Summarizing, we have proved:

3.3. TheOREM. Let $G$ be a direct indecomposable nonabelian group and suppose $Z(G)$ is finite. Let $n \in \mathbb{N}$ and put $S:=\operatorname{Hom}(G, Z(G))$. Then

$$
\operatorname{Aut}(G \times \underset{n}{\cdots} \times G) \cong(\operatorname{Aut}(G)) \underset{S}{\varsigma_{S}} \text {. }
$$

If $G$ satisfies the additional condition $\operatorname{Hom}(G, Z(G))=0$ (which is in the finite case equivalent to $\left.\left(\left|G / G^{\prime}\right|,|Z(G)|\right)=1\right)$, our Theorem yields via (45) the well-known statement:

$$
\operatorname{Aut}(G \times \underset{n}{\cdots} \times G) \cong(\operatorname{Aut}(G)) \backslash \mathfrak{S}_{n} .
$$




\section{REFERENCES}

[1] H. Fitting, Über die direkten Produktzerlegungen einer Gruppe in direkt unzerlegbare Faktoren, Math. Z., 39 (1935), 16-30.

[2] H. Fitting, Über den Automorphismenbereich einer Gruppe, Math. Ann., 114 (1937), 84-98.

[3] N. Jacobson, Structure of Rings, AMS Coll. Publ. vol. XXXVII (1956).

Received March 8, 1984.

UNIVERSITÄT KIEL

OLSHAUSENSTR. 40-60

2300 KiEL 1, W. GERMANY 


\section{PACIFIC JOURNAL OF MATHEMATICS EDITORS}

\author{
V. S. VARADARAJAN (Managing Editor) \\ University of California \\ Los Angeles, CA 90024 \\ Hebert Clemens \\ University of Utah \\ Salt Lake City, UT 84112 \\ Charles R. DePrima \\ California Institute of Technology \\ Pasadena, CA 91125
}

R. FINN

Stanford University

Stanford, CA 94305

HermanN FlaschKa

University of Arizona

Tucson, AZ 85721

RAMESH A. GANGOLlI

University of Washington

Seattle, WA 98195

ROBION KIRBY

University of California

Berkeley, CA 94720

ASSOCIATE EDITORS
R. ARENS
E. F. BECKENBACH
B. H. NEUMANN
F. WOLF
K. YoshidA (1906-1982)

C. C. Moore

University of California

Berkeley, CA 94720

H. SAMELSON

Stanford University

Stanford, CA 94305

HAROLD STARK

University of California, San Diego

La Jolla, CA 92093

\section{SUPPORTING INSTITUTIONS}

UNIVERSITY OF ARIZONA

UNIVERSITY OF BRITISH COLUMBIA

CALIFORNIA INSTITUTE OF TECHNOLOGY

UNIVERSITY OF CALIFORNIA

MONTANA STATE UNIVERSITY

UNIVERSITY OF NEVADA, RENO

NEW MEXICO STATE UNIVERSITY

OREGON STATE UNIVERSITY
UNIVERSITY OF OREGON

UNIVERSITY OF SOUTHERN CALIFORNIA

STANFORD UNIVERSITY

UNIVERSITY OF HAWAII

UNIVERSITY OF TOKYO

UNIVERSITY OF UTAH

WASHINGTON STATE UNIVERSITY

UNIVERSITY OF WASHINGTON 


\section{Pacific Journal of Mathematics}

Vol. 122, No. $1 \quad$ January, 1986

Michael James Cambern, Near isometries of Bochner $L^{1}$ and $L^{\infty}$ spaces ....1 Kun Soo Chang, Gerald William Johnson and David Lee Skoug, The

Feynman integral of quadratic potentials depending on two time

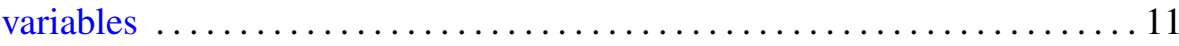

Robert Coleman, One-dimensional algebraic formal groups $\ldots \ldots \ldots \ldots \ldots 35$

Alberto Collino, The Abel-Jacobi isomorphism for the cubic fivefold .......43

N. J. Dev and S. S. Khare, Finite group action and vanishing of $N_{*}^{G}[F] \ldots 57$

Harold George Diamond and Jeffrey D. Vaaler, Estimates for partial sums

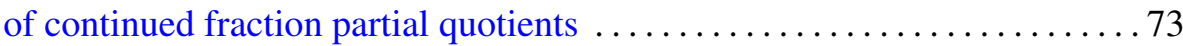

Kenneth R. Goodearl, Patch-continuity of normalized ranks of modules

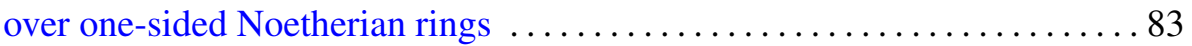

Dean Robert Hickerson and Sherman K. Stein, Abelian groups and

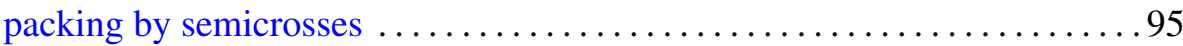

Karsten Johnsen and Harmut Laue, Fitting structures $\ldots \ldots \ldots \ldots \ldots 11$

Darren Long, Discs in compression bodies . ................... 129

Joseph B. Miles, On the growth of meromorphic functions with radially distributed zeros and poles ........................... 147

Walter Volodymyr Petryshyn, Solvability of various boundary value problems for the equation $x^{\prime \prime}=f\left(t, x, x^{\prime}, x^{\prime \prime}\right)-y \ldots \ldots \ldots \ldots \ldots . \ldots 169$

Elżbieta Pol, The Baire-category method in some compact extension problems

Masami Sakai, A new class of isocompact spaces and related results 211

Thomas Richard Shemanske, Representations of ternary quadratic forms and the class number of imaginary quadratic fields ..

Tsuyoshi Uehara, On class numbers of cyclic quartic fields 